\title{
Excitation of the FUV Io tail on Jupiter: Characterization of the electron precipitation
}

\author{
J.-C. Gérard, J. Gustin, and D. Grodent \\ Laboratoire de Physique Atmosphérique et Planétaire, Université de Liège, Belgium \\ P. Delamere \\ Laboratory for Atmospheric and Space Physics, University of Colorado, Boulder, Colorado, USA
}

J. T. Clarke

Center for Space Physics, Boston University, Boston, Massachusetts, USA

Received 27 March 2002; revised 28 May 2002; accepted 8 August 2002; published 22 November 2002.

[1] Spectral observation of both polar regions of Jupiter in the far ultraviolet (FUV) obtained with the Space Telescope Imaging Spectrograph (STIS), on board the Hubble Space Telescope from July 1997 to January 2001 have been combined with FUV images to map the FUV color ratio along the STIS slit. Spatially resolved spectra of the aurora carried at $\sim 12 \AA$ resolution have been used to determine the amount of methane absorption as measured by the FUV color ratio of the Io magnetic footprint and its trailing tail. It is found that the absorption is systematically less than in the main polar aurora, indicating a higher altitude source region. The color ratio of the north tail is shown to slowly decrease downstream from the footprint. The combination of these spectral data with a two-stream model of the interaction of energetic electrons with the Jovian thermosphere indicates that the mean energy of the electrons creating the north FUV emission ranges from $\sim 55 \mathrm{keV}$ at the Io footprint to $\sim 40 \mathrm{keV}, 20$ degrees downstream in the tail. In parallel, the incident electron energy flux drops by a factor $\sim 6$ over the same angular distance. These observations are consistent with the steady state slippage picture where the subcorotating flux tube is accelerated very slowly up to corotation owing to the nonideal coupling. It is argued that small deviations from corotation can supply sufficient energy to fuel the observed auroral emissions. It is suggested that the parallel electric field accelerating electrons out of the flux tube only moderately depends on the time elapsed since the contact with Io, although the mapping between a point in the tail and Io is very uncertain in the presence of magnetic field line slippage. INDEX TERMS: 2704 Magnetospheric Physics: Auroral phenomena (2407); 6220 Planetology: Solar System Objects: Jupiter; 6218 Planetology: Solar System Objects: Jovian Satellites; 2708 Magnetospheric Physics: Current systems (2409); KEYWORDS: Io, flux tube, ultraviolet spectroscopy, Jupiter, electron energy, slippage

Citation: Gerard, J.-C., J. Gustin, D. Grodent, P. Delamere, and J. T. Clarke, Excitation of the FUV Io tail on Jupiter: Characterization of the electron precipitation, J. Geophys. Res., 107(A11), 1394, doi:10.1029/2002JA009410, 2002.

\section{Introduction}

[2] The interaction between Jupiter and Io has been extensively studied since the discovery that Jupiter's decametric emissions are controlled by the position of Io along its orbit. A current of $\sim 5 \times 10^{6}$ A was observed to flow between the two bodies by Voyager 1 [Acuña et al., 1981]. It was initially explained by a unipolar inductor model where the motion of Io across the Jovian magnetic field lines generate $\mathrm{a} \sim 500 \mathrm{keV}$ electric potential driving currents along Jupiter's field lines. The current circuit is closed in Jupiter's ionosphere and flows back to Io. Measurements suggest instead that the interaction is primarily controlled by mass loading [Goertz, 1980; Saur et al., 1999; Russell and Huddleston, 2000] which drives strong parallel currents along the flux tubes of the associated Alfvenic disturbance [Neubauer, 1980; Belcher et al., 1981]. Another theory involves the generation of Alvenic waves propagating away from Io to Jupiter [Neubauer, 1980; Belcher et al., 1981]. The existence of an Alfvenic current is consistent with the low flow speed of the flux tubes in Io's wake measured by Galileo [Frank et al., 1996]. Intense field-aligned lowenergy electron beams toward and from Jupiter were observed with the Galileo spacecraft by Frank and Paterson [1999]. These beams of low-energy (several hundred eV) electrons aligned along the magnetic field were detected in 
the wake of Jupiter torus plasma flows past Io. The power carried by these beams was estimated of the order of $3 \times$ $10^{10}$ W. Williams et al. [1999] measured energy fluxes at electron energies above $15 \mathrm{keV}, 50$ to 100 times less than those associated with the low-energy component.

[3] This general picture was dramatically confirmed by the discovery of an infrared spot near the foot of the Io flux tube [Connerney et al., 1993]. The feature moves across the Jovian disc as expected from the Io orbital motion. Bright far ultraviolet auroral emissions associated with the footprint were also observed with the Hubble Space Telescope cameras [Prangé et al., 1998; Clarke et al., 1996]. The FUV Io footprint emission is characterized by a typical brightness of $\sim 30-280 \mathrm{kR}$ in the WFPC2 passband averaged over a $0.5 \times 0.5 \mathrm{arc} \mathrm{sec}^{2}$ area. Its location is coincident in latitude with the projection of the footprint mapped along the unperturbed Jovian magnetic field. It shows a longitudinal lead angle in the direction of Io's orbital motion from the instantaneous magnetic projection varying from $0^{\circ}$ to $10^{\circ}$, depending on the System III longitude. The total power associated with the Io footprint emission is estimated from $6 \times 10^{9} \mathrm{~W}$ to $5 \times 10^{10} \mathrm{~W}$. Crary [1997] proposed that the source of the infrared and FUV Io footprints is a precipitated electron beam generated by the wave's parallel electric field through a process of repeated Fermi accelerations. An alternative mechanism was proposed by Shaponish-Nikov et al. [2001] assuming that the emission from the footprint is excited inside the Jovian atmosphere by collisions of ionospheric particles with the ambient gas [Nishida and Watanabe, 1981]. The motion would be induced by the Ampère force owing to the interaction of the electric current within the flux tube with its own magnetic field.

[4] The sensitive Space Telescope Imaging Spectrograph (STIS) on board the HST shows a complex, sometimes doubly peaked time-varying structure of the auroral Io footprint emissions [Clarke et al., 2002]. Images show a long trailing tail extending eastward from the instantaneous mapping of Io for at least $100^{\circ}$ in longitude along the magnetic footprint of Io's orbit. The brightness decreases downstream with a characteristic distance of typically $5^{\circ}-$ $10^{\circ}$ longitude. This region would correspond to plasma picked up from Io by the corotating magnetic field of Jupiter. Possible explanations for this extended tail include departure from corotation or multiply reflecting Alfvén waves. However, a large number of reflections are needed to extend $100^{\circ}$ downstream and the intensity of the residual waves would presumably drop to a negligible level on a shorter spatial scale [Clarke et al., 2002]. So far, no clear picture has emerged as to the cause of the extended Io footprint tail emission, and additional observations are clearly needed to further constrain models. STIS-HST spatially resolved spectroscopy offers an opportunity to measure the FUV color ratio at different locations along the Io trailing tail. This ratio is an indicator of the amount of absorption by hydrocarbon and thus of the characteristic energy of the energetic particles causing the Io tail emission. Coupled with an electron transport model, the color ratio may be used to determine the characteristic energy of the precipitation. A first glance was provided by Dols et al.'s [2000] study of the FUV color ratio associated with the Io footprint. A mean energy of $60 \mathrm{keV}$ was obtained for the Io spot located at $65^{\circ} \mathrm{S}$. In this study we analyze the FUV color ratio associated with the Io footprint and tail in both hemispheres. In particular we examine whether the decreasing intensity downstream is associated with a drop of the electron flux or mean energy or a combination of both.

\section{Observations}

[5] Far ultraviolet auroral spectra were obtained with the Space Telescope Imaging Spectrograph from July 1997 to January 2001. The spatially resolved spectra were imaged on the FUV MultiAnode Microchannel Array (MAMA) photon counting detector providing a $1024 \times 1024$ pixels image. The G140L grating combined with the $26 \times 0.5 \operatorname{arcsec}^{2}$ long-slit provided spectra in the 1100-1700 $\AA$ range with $\sim 12 \AA$ resolution. The instrument was operated in the TIMETAG mode and provided an event stream with fast time resolution. The spectra taken in each HST orbit were preceded and followed by auroral images using the $\mathrm{SrF}_{2}$ filter rejecting the HI Lyman- $\alpha$ emission or in the MAMA clear mode transmitting light from 1150 to $1780 \AA$. A total of 25 spectra in the Northern Hemisphere and 13 spectra in the Southern Hemisphere were analyzed. Out of the 38 spectra, 15 showed a measurable signature of the Io trail. In all other observations the Io trail was either eastward of the STIS slit or too far westward to measure a detectable brightness. The slit was usually positioned close to the central meridian, but it was moved toward the morning or the evening limbs to probe different regions of the Io tail or auroral oval. Processing of the STIS spectra and the background subtraction were described by Gustin et al. [2002]. The count rate was converted into physical units of kiloRayleigh (kR) and erg $\mathrm{cm}^{-2} \mathrm{~s}^{-1}$, based on the absolute instrumental response, in-flight calibrations and synthetic $\mathrm{H}_{2}$ spectra.

[6] Table 1 lists the observation dates and times of the Io trail, together with the exposure length, hemisphere, central meridian and Io longitudes (System III) and approximate distance downstream of the Io Footprint. The latitudinally integrated trail brightness and the color ratio were determined for each spectrum. The FUV color ratio

$$
\mathrm{CR}=\frac{\mathrm{I}(1550-1620 \AA)}{\mathrm{I}(1230-1300 \AA)}
$$

provides a measure of the attenuation of the $\mathrm{H}_{2}$ emission below $1350 \AA$ by methane overlying the emission layer. Using a model, it can be used to determine the penetration of the incident electrons relative to the $\mathrm{CH}_{4}$ homopause. The projection of the $26 \times 0.5 \operatorname{arcsec}^{2}$ slit is first overlaid on the image taken closest in time before or after each spectrum. We extract the brightness curve along the position of the slit on the basis of the nominal telescope pointing. The intensity distribution is then compared with the intensity measured along the slit, and we allow the slit center to be displaced by up to 1 arcsec until a good match is obtained between the auroral features seen in the image and the spectrum. This position is used to define the intersection of the STIS slit with the Io trail. Figure 1 shows an example of a FUV image of the north polar region and the position of the STIS slit during spectral observations carried 12 min later. The traces of intensity variation along the slit in the two spectral regions clearly show the signature of the two auroral crossings and of the Io footprint. The 
Table 1. Characteristics of the Io Tail Spectral Observations With STIS

\begin{tabular}{|c|c|c|c|c|c|c|c|}
\hline Observation & Date & $\begin{array}{c}\text { UT, } \\
\text { hh:mm }\end{array}$ & $\begin{array}{c}\text { Exposure, } \\
\text { sec }\end{array}$ & $\mathrm{N} / \mathrm{S}$ & $\begin{array}{c}\text { CML, } \\
\lambda_{\text {III }}, \text { deg }\end{array}$ & $\begin{array}{c}\text { Io Footprint } \\
\text { Longitude, } \lambda_{\text {III }} \text {, deg }\end{array}$ & $\begin{array}{l}\text { Distance From } \\
\text { Footprint, deg }\end{array}$ \\
\hline 1 & $22 \mathrm{Feb} 00$ & $16: 32$ & 240 & $\mathrm{~N}$ & 118 & 151 & 10.5 \\
\hline 2 & $22 \mathrm{Feb} 00$ & $16: 40$ & 240 & $\mathrm{~N}$ & 122 & 152 & 4 \\
\hline 3 & 22 Feb 00 & $16: 47$ & 240 & $\mathrm{~N}$ & 127 & 155 & 0 \\
\hline 4 & 14 Dec 00 & $12: 37$ & 630 & $\mathrm{~N}$ & 228 & 178 & 10 \\
\hline 5 & 20 Jan 01 & $15: 58$ & 260 & $\mathrm{~N}$ & 160 & 152 & 0 \\
\hline 6 & 20 Jan 01 & $16: 08$ & 260 & $\mathrm{~N}$ & 166 & 154 & 20 \\
\hline 7 & 20 Jan 01 & $16: 17$ & 260 & $\mathrm{~N}$ & 172 & 157 & 20 \\
\hline 8 & 21 Jan 01 & $22: 49$ & 260 & $\mathrm{~N}$ & 199 & 240 & 15 \\
\hline 9 & 20 Sep 97 & $14: 36$ & 400 & $\mathrm{~S}$ & 107 & 122 & 6 \\
\hline 10 & 8 Aug 99 & $11: 46$ & 260 & $\mathrm{~S}$ & 19 & 46 & 0 \\
\hline 11 & 8 Aug 99 & $11: 54$ & 260 & $\mathrm{~S}$ & 24 & 47 & 0 \\
\hline 12 & 8 Aug 99 & $12: 02$ & 260 & $\mathrm{~S}$ & 29 & 48 & 5 \\
\hline 13 & 8 Aug 99 & $13: 10$ & 200 & $\mathrm{~S}$ & 70 & 74 & 2 \\
\hline 14 & 8 Aug 99 & $13: 17$ & 200 & $\mathrm{~S}$ & 74 & 75 & 0 \\
\hline 15 & 8 Aug 99 & $13: 24$ & 200 & $\mathrm{~S}$ & 78 & 90 & 0 \\
\hline
\end{tabular}

ratio of the two emissions is significantly less on the footprint than in the aurora.

\section{Spectral Data}

[7] We first examine a summary plot of the complete data set of auroral STIS spectra. Figure 2 shows the variation of the color ratio as a function of dates for three different features of the north Jovian aurora. The color ratios of the auroral main oval, polar cap, and Io tail show distinct behaviors. The main oval exhibits large variations ranging from 1.4 to 10.4 . The polar cap ratio is also highly variable, although no values higher than 6.7 are observed. In comparison, the Io trail color ratios are remarkably constant and low in comparison with the other regions. The measured color ratios for all footprint and tail spectra are listed in Table 2. An emission source region located above the methane layer would yield a color ratio of 1.1, lower than any of the observed values. Five of the 15 observations were made directly on the Io footprint, but they are not signifi- cantly different from the ratio of the trailing tail's portion intercepted by the STIS slit. Individual scans along the Io tail (cases $1-2-3$ and 5-6) indicate that the color ratio slightly drops as the slit is moved downstream.

[8] Although our sample is limited, no systematic variation of the color ratio is observed with local time or with the sub-Io SIII longitude that is the location in the plasma torus or the intrinsic brightness of the unabsorbed signal. However, a slight decreasing trend appears with the distance downstream in the tail. Figure 3 shows the statistical variation of the color ratio as a function of the (eastward) distance from the Io footprint. It appears to decrease linearly with a slope of 0.019 /degree of longitude with a $\mathrm{R}^{2}$ Pearson coefficient of 0.66 . Error bars on the individual data points of color ratio are of the order of $10 \%$. The data from the south show no such clear trend with a larger dispersion than in the north. No data are available at angular distances larger than $20^{\circ}$, owing to the limited sample of HST-STIS spectral observations and the decreasing brightness of the Io trail. We now describe how the measured color ratios may

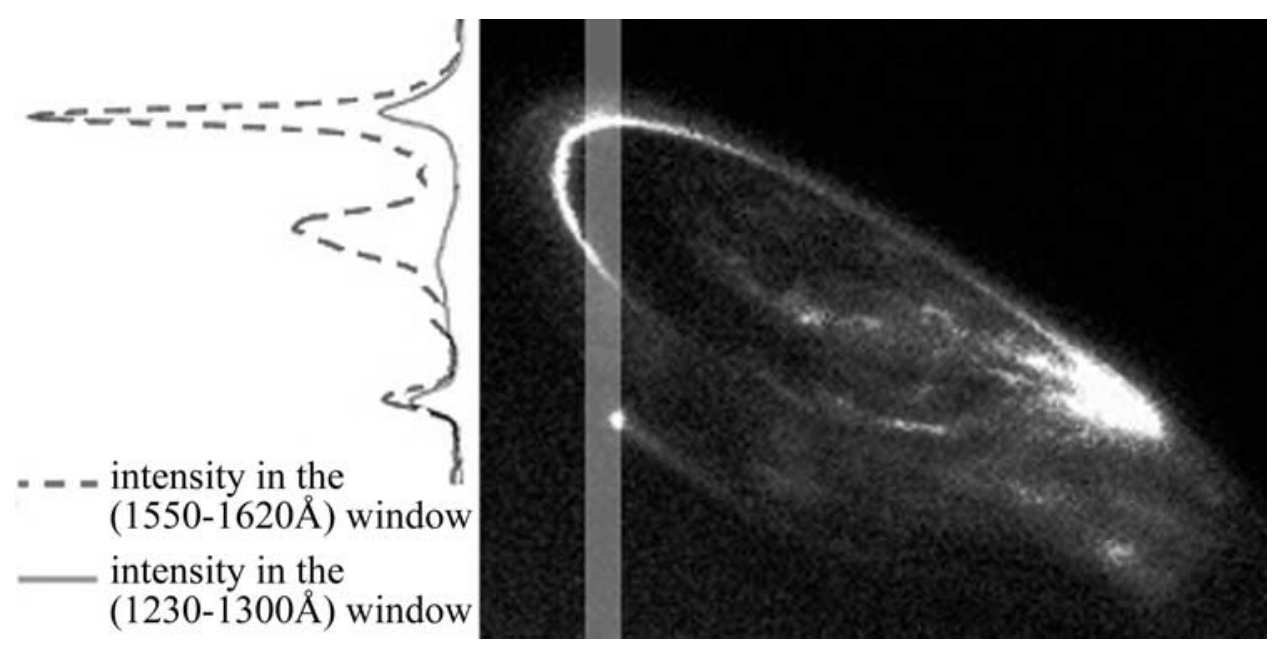

Figure 1. Geometry of the spectral observations of the Io footprint. The image was obtained on 22 February 2000 approximately 12 min before the G140L spectrum (case 3 in Table 1). The position of the STIS slit is indicated as a lighter blue line. The intensity variations measured along the slit in two spectral regions (one absorbed by methane, one not) are shown on the left. See color version of this figure at back of this issue. 


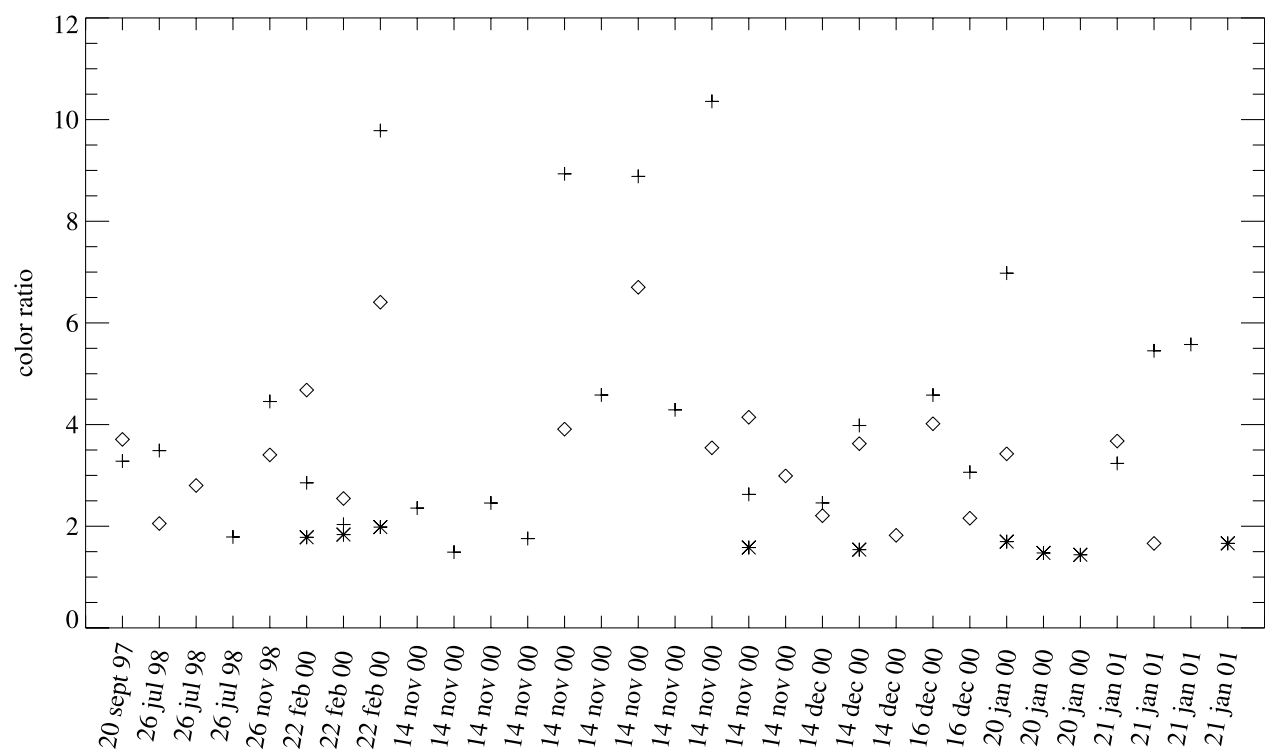

date

Figure 2. FUV color ratio determined from the STIS spectra obtained between July 1997 and January 2001. The symbols correspond to different regions of the Jovian aurora: Io footprint and tail (star), main auroral oval (plus), and polar cap (diamond). An extreme case with a color ratio exceeding 20 in the main oval measured on 21 September 1999 is not included.

be converted into characteristic energies of precipitating electrons.

\section{Model and Electron Energy Determination}

[9] Using an atmospheric model of the Jovian atmosphere, the FUV auroral color ratio may be used to determine the mean energy of the precipitated electrons. In a similar way, one may assume that the Io footprint is excited by electrons accelerated along the magnetic field lines connecting Io and the Jovian ionosphere. In this case, the (nearly constant) color ratio combined with the measured brightness will provide an estimate of the electron flux and mean energy. The model used to calculate the interaction of the energetic electrons with the Jovian atmosphere was described in detail by Grodent et al. [2001]. This model couples a two-stream electron transport model of energy deposition with a one-dimensional thermal conduction model including thermal cooling to calculate a self-consistent thermal structure adapted to the auroral upper atmosphere. The two-stream code calculates the flow and redistribution of the electron energy as a function of altitude from atomic and molecular processes by means of discrete energy bins. Once the electron fluxes are calculated, the rates of ionization, dissociation, heating, and emission processes can be determined. In the version used for this study the thermal structure is not allowed to adjust to the local thermal balance. The neutral atmosphere is taken from Gladstone et al.'s [1996] NEB model. The gravity acceleration is adapted to a latitude of $60^{\circ} \mathrm{N}$. The methane vertical distribution is obtained by combining the lower boundary-mixing ratio from Drossart et al. [1993] with the solution of the one-dimensional diffusion equation. The eddy coefficient is set to $1.4 \times 10^{6} \mathrm{~cm}^{2} \mathrm{~s}^{-1}$. Synthetic $\mathrm{H}_{2}$ spectra are generated as described by Dols et al. [2000]. The emerging intensity at each wavelength is calculated from the weighted sum of the spectra, including hydrocarbon absorption when present. The final emerging spectrum is smoothed by the instrumental line spread function and the color ratio $\mathrm{CR}$ is calculated for a given initial electron energy spectrum. In the absence of observation on the shape of this energy spectrum, we use a Maxwellian energy distribution characterized by a mean electron energy $\langle\mathrm{E}\rangle=2 \mathrm{E}_{0}$, a number flux $\mathrm{N}_{0}$, and an energy flux $\mathrm{F}_{0}=2 \mathrm{~N}_{0} \mathrm{E}_{0}$, where $\mathrm{E}_{0}$ is the characteristic energy. The calculated relationship between the color ratio and $\langle\mathrm{E}\rangle$ for a view angle of $60^{\circ}$ is shown in Figure 4.

Table 2. Brightness and Electron Characteristics Measured Along the Io Tail

\begin{tabular}{cccc}
\hline Observation & $4 \pi \mathrm{I}^{\mathrm{a}}, \mathrm{kR}$ & Color Ratio & $\langle\mathrm{E}\rangle^{\mathrm{b}}, \mathrm{keV}$ \\
\hline 1 & 33 & 1.78 & 54 \\
2 & 82 & 1.83 & 56 \\
3 & 108 & 1.98 & 61 \\
4 & 148 & 1.54 & 46 \\
5 & 161 & 1.70 & 52 \\
6 & 27 & 1.47 & 43 \\
7 & 21 & 1.44 & 41 \\
8 & 12 & 1.66 & 50 \\
9 & 60 & 2.16 & 66 \\
10 & 146 & 1.53 & 45 \\
11 & 278 & 1.77 & 44 \\
12 & 152 & 1.51 & 44 \\
13 & 234 & 1.74 & 53 \\
14 & 568 & 2.28 & 69 \\
15 & 673 & 2.16 & 66 \\
\hline
\end{tabular}

${ }^{\mathrm{a}} 4 \pi \mathrm{I}$ refers to the total brightness of the $\mathrm{H}_{2}$ emission from $\mathrm{B}$ and $\mathrm{C}$ states.

${ }^{\mathrm{b}}\langle\mathrm{E}\rangle$ is the estimated average electron energy assuming an initial Maxwellian energy distribution. 


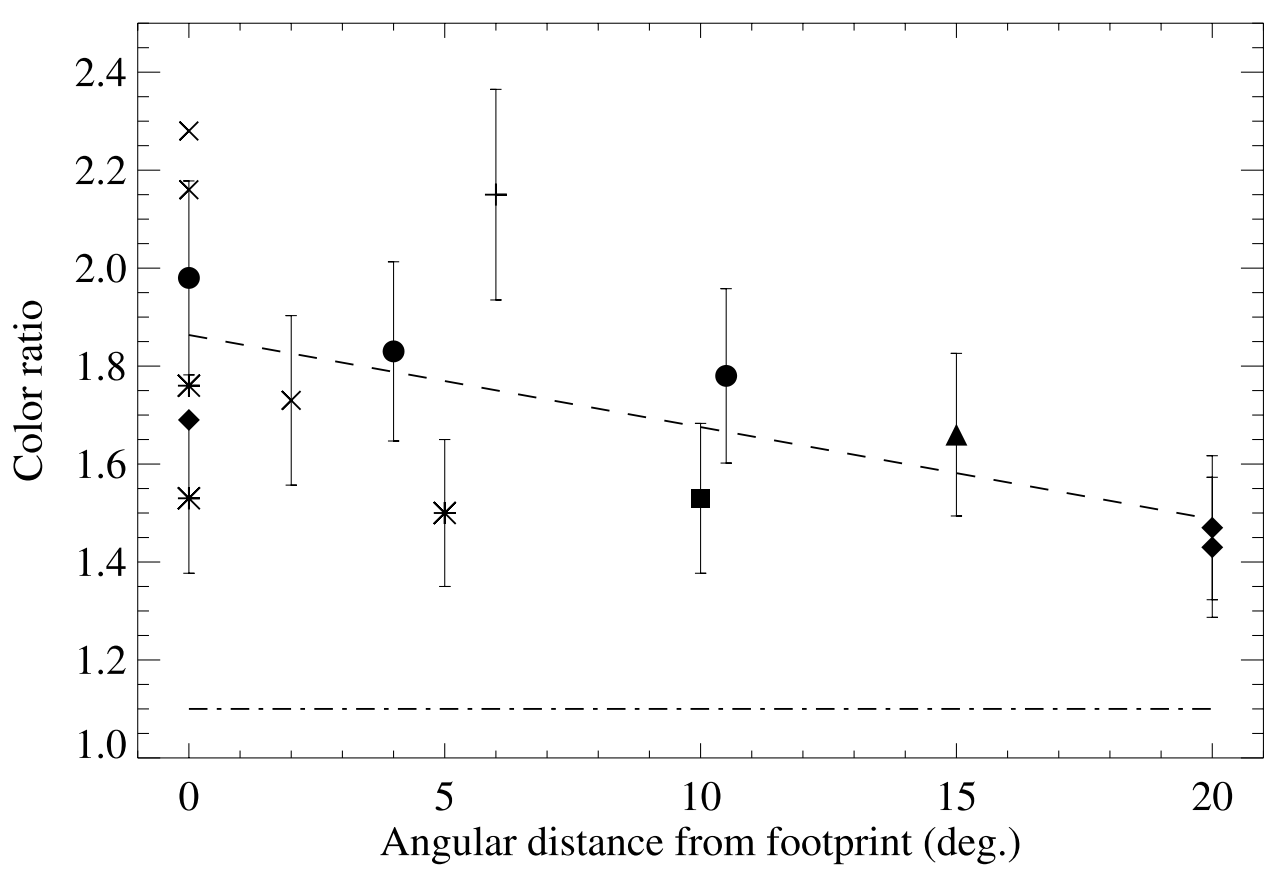

Figure 3. Variation of the color ratio observed along the Io trailing tail as a function of the angular distance from the footprint. Identical symbols are used for measurements obtained within one HST orbit. The closed symbols (circle, diamond, square, triangle) correspond to north data, the other symbols $(*,+$, $\mathrm{X})$ to the south. The dashed line is the linear regression fitted to the north data and the dot-dashed horizontal line indicates the color ratio with no hydrocarbon absorption.

[10] Table 2 summarizes the main quantitative features associated with each footprint or trailing tail crossing. It lists the emission brightness, the color ratio, and the mean electron energy $\langle\mathrm{E}\rangle$ for each observation. Average electron energies associated with the footprint and trail range between $\sim 40$ and $\sim 70 \mathrm{keV}$, with a mean value of $48 \mathrm{keV}$. As mentioned before, the trend of decreasing $\mathrm{CR}$ and $\langle\mathrm{E}\rangle$ is present both in the two individual scans in the north and in the statistical plot. The $\mathrm{H}_{2}$ brightness refers to the total unabsorbed emission from 114 to $170 \mathrm{~nm}$, which includes most of the Lyman and Werner band emissions. Our model and earlier studies [Gérard and Singh, 1982; Waite et al., 1983; Grodent et al., 2001] indicate that the efficiency of the electron energy conversion into B and C states excita-

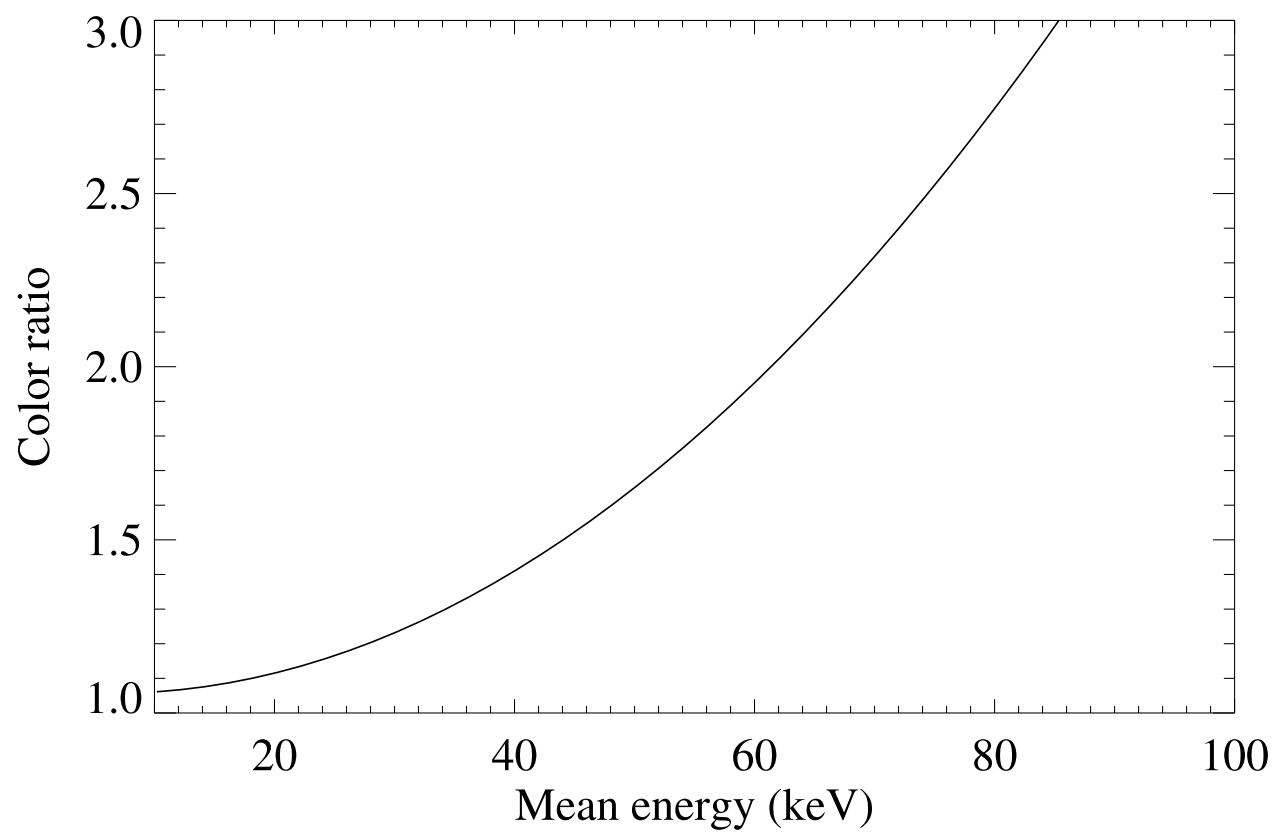

Figure 4. Calculated variation of the FUV color ratio as a function of the mean electron energy for a Maxwellian distribution of the primary electrons at the top of the atmosphere and a view angle of $60^{\circ}$. 
tion is close to $10 \mathrm{kR} /$ incident erg $\mathrm{cm}^{-2} \mathrm{~s}^{-1}$ for primary energies between 10 and $100 \mathrm{keV}$. This factor may be used to derive the energy flux carried by the incident energetic electrons. The precipitated electron energy flux typically drops from $33 \mathrm{~mW} / \mathrm{m}^{2}$ at the footprint to $6 \mathrm{~mW} / \mathrm{m}^{2}, 20$ degrees downstream. It clearly decreases more rapidly than $\langle E\rangle$ with distance from the footprint.

[11] Combining both results, it appears that the drop of brightness along the Io trail is due $\sim 80 \%$ to a decrease of the electron number flux rather than a softening of the electron mean energy. The timetag mode makes it possible to observe the time evolution of the observed spectral features. In some cases, variations of brightness by a factor of two are observed but the associated color ratio does not show any significant variation. It may thus be concluded that the corresponding electron energy also remains constant. This is an important constraint to understand the processes leading to the excitation of the Io footprint and its extended tail.

\section{Discussion}

[12] The determination of the electron energy from the color ratio relies on the atmospheric model. If the vertical distribution of methane is significantly different from what we assumed, the relationship between the color ratio and the electron energy would be altered. Such a possibility could take place if the energy deposition causes strong upwelling bringing methane-richer gas above the quiet homopause [Livengood et al., 1990]. For example, Grodent et al. [2001] showed that if the eddy-mixing coefficient is one order of magnitude higher than used here, the methane homopause level rises by $\sim 66 \mathrm{~km}$. The increased absorption for a given observed color ratio implies a characteristic electron energy less than with the standard model. Sensitivity tests have shown that a typical color ratio of 1.7 reached for a mean electron energy of $52 \mathrm{keV}$ in our standard model (Figure 4) would correspond to $38 \mathrm{keV}$ if the eddy mixing were twice as vigorous. Our results clearly show that the precipitation associated with the Io trailing tail is softer than that usually observed in the main auroral oval and in the polar cap. Statistically, the mean energy in the north drops from 55 to $42 \mathrm{keV}$ over $20^{\circ}$ of longitude, that is, on the order of $40 \mathrm{~min}$ after the passage of Io. As discussed by Clarke et al. [1998] and confirmed by Table 2, more electrons are precipitated in the south footprint than in the north, presumably as a consequence of the weaker magnetic field at southern high latitudes.

[13] Crary's [1997] model of the electron beam producing the Io footprint predicts an estimated power of 0.5 to $1.5 \times 10^{11} \mathrm{~W}$ and an average electron energy of $\sim 75 \mathrm{keV}$. These values are close to the values derived for the Io footprint. This model assumes that electrons of the flux tube are accelerated along the field lines by the parallel electric field generated by the Alfvenic waves. It can be speculated that such multiply reflected Alfvèn waves may also account for the periodicities in the decametric (DAM) emission [Wilkinson, 1998] and possibly the arc structures observed in the non-DAM emission [Goldstein and Thieman, 1981].

[14] At this point, no numerical model of the extended trailing tail can directly be tested against these observations.
However, the problem has been discussed in the context of general magnetosphere-ionosphere (M-I) coupling where the coupling is controlled by aurora-like parallel potentials [Mauk et al., 2002]. The coupling between Iogenic plasma and Jupiter's ionosphere is essentially a momentum transfer problem. In the Io reference frame there is a region of stagnant plasma flow immediately behind Io [Frank et al., 1996]. For ideal plasma flow the velocity shear between the stagnant wake and the adjacent high-speed plasma generates field-aligned currents associated with the Alfvenic disturbance. Momentum is transferred to the stagnant wake from the ambient corotating plasma via Alfven waves and momentum conservation guarantees that on average the mass-loaded flux tube will be subcorotating until full coupling to Jupiter's ionosphere can be achieved. Ideal coupling is only possible in the absence of a field-aligned potential drop, which is clearly not possible given the observed auroral emissions. Parallel electric fields lead to nonideal plasma flow, or "slippage," limiting the fieldaligned currents and hence the momentum transfer rates [Schinder et al., 1991, Delamere et al., 2000]. As a result, the Io perturbed flux tube will be accelerated up to corotation on a much longer timescale.

[15] If we assume that the extended tail emissions represent a quasi-steady state configuration, a simple estimate can be made of the corotational lag of the Io perturbed flux tube. The potential contours are closed by the parallel electric fields and in steady state, $\nabla \times \mathbf{E}=0$. Consequently, the field aligned potential drop is roughly equal to the perpendicular potential drop above the acceleration region. In the corotating reference frame, the velocity that is necessary to provide a $40 \mathrm{kV}$ potential across one Io diameter (dimension of stagnated flow region) is $\varphi /\left(2.0 \quad B_{0} \quad R_{I o}\right) \sim 5.4 \mathrm{~km} / \mathrm{s}$. This represents a lower limit as the entire field-aligned potential drop is assumed to be in the upward current region. Potential drops in the downward current region and across Jupiter's ionosphere would tend to increase the estimated deviation from corotation.

[16] Thus small deviations from corotation can supply sufficient energy to fuel the observed auroral emissions. It should be noted, however, that the relationship between the distance from the footprint and the time elapsed since a field line contacts Io is complex, since the degree of slippage is unknown. It is therefore possible that points down the tail could still be in contact with Io.

[17] Our observations indicate that the drop in brightness along the trail is due to a decrease in electron number flux rather than a softening of the electron mean energy. This is consistent with the steady state slippage picture where the subcorotating flux tube is accelerated very slowly up to corotation due to the nonideal coupling. Thus the perpendicular potential drop remains roughly constant due to negligible momentum transfer. The softening of the electron flux may be due to a broadening of the acceleration region.

[18] Acknowledgments. This work is based on observations with the NASA/ESA Hubble Space Telescope obtained at the Space Telescope Science Institute (STScI), which is operated by the AURA, Inc. for NASA. JCG and DG acknowledge support from the Belgian Fund for Scientific Research and the PRODEX program of ESA. This research was also supported by grants from the Space Telescope Science Institute and from NASA to the University of Michigan. 
[19] Arthur Richmond thanks Frank Crary and Louis A. Frank for their assistance in evaluating this paper.

\section{References}

Acuña, M. H., F. M. Neubauer, and N. F. Ness, Standing Alfvén wave current system at Io: Voyager 1 observations, J. Geophys. Res., 86, 8513, 1981

Belcher, J. W., et al., Plasma observations of the Alfvén wave generated by Io, J. Geophys. Res., 86, 8508, 1981.

Clarke, J. T., et al., Far-ultraviolet imaging of Jupiter's aurora and the Io "footprint", Science, 274, 404, 1996.

Clarke, J. T., et al., Hubble Space Telescope imaging of Jupiter's UV aurora during the Galileo orbiter mission, J. Geophys. Res., 103, 20,217, 1998.

Clarke, J. T., et al., Ultraviolet emissions from the magnetic footprints of Io, Ganymede, and Europa on Jupiter, Nature, 415, 997, 2002.

Connerney, J. E. P., et al., Images of excited $\mathrm{H}_{3}{ }^{+}$at the foot of the Io flux tube in Jupiter's atmosphere, Science, 262, 1035, 1993.

Crary, F. J., On the generation of an electron beam by Io, J. Geophys. Res., 102, 37-49, 1997.

Delamere, P. A., H. C. Stenbaek-Nielsen, D. W. Swift, and A. Otto, Momentum transfer in the Combined Release and Radiation Effects Satellite plasma injection experiments: The role of parallel electric fields, Phys. Plasmas, 7, 3771, 2000.

Dols, V., J. C. Gérard, V. Clarke, J. Gustin, and D. Grodent, Diagnostics of the Jovian aurora deduced from ultraviolet spectroscopy: Model and GHRS observations, Icarus, 147, 251, 2000.

Drossart, P., B. Bézard, S. K. Atreya, J. Bishop, J. H. Waite Jr., and D. Boice, Thermal profiles in the auroral regions of Jupiter, J. Geophys. Res., 98, 18,803, 1993.

Frank, L. A., and W. R. Paterson, Intense electron beams observed at Io with the Galileo spacecraft, J. Geophys. Res., 104, 28,657, 1999.

Frank, L. A., et al., Plasma observations at Io with the Galileo spacecraft, Science, 274, 394, 1996.

Gérard, J. C., and V. Singh, A model of energetic electrons and EUV emission in the Jovian and Saturnian atmospheres and implications, J. Geophys. Res., 87, 4525, 1982.

Goertz, C. K., Io's interaction with the plasma torus, J. Geophys. Res., 85, 2949,1980

Goldstein, M. L., and J. T. Thieman, The formation of arcs in the dynamic spectra of Jovian decameter burst, J. Geophys. Res., 86, 8569, 1981.

Grodent, D., J. H. Waite Jr., and J. C. Gerard, A self-consistent model of the Jovian auroral thermal structure, J. Geophys. Res., 106, 12,933, 2001.

Gustin, J., D. Grodent, J. C. Gérard, and J. T. Clarke, Spatially resolved far ultraviolet spectroscopy of the Jovian aurora, Icarus, 156, 91, 2002.

Mauk, R. H., B. J. Anderson, and R. M. Thorne, Magnetosphere-ionosphere coupling at Earth, Jupiter, and beyond, Comparative Aeronomy, Geophys. Monogr. Ser, vol. 130, edited by M. Mendillo, A. Nagy, and J. H. Waite, AGU, Washington, D.C., 2002.

Neubauer, F. M., Nonlinear standing Alfven wave current system at Io: Theory, J. Geophys. Res., 85, 1171, 1980.

Nishida, A., and Y. Watanabe, Joule heating of the Jovian ionosphere by corotation enforcement currents, J. Geophys. Res., 86, 9945, 1981.

Prangé, R., D. Rego, L. Pallier, J. Connerney, P. Zarka, and J. Queinnec, Detailed study of FUV Jovian auroral features with the post COSTAR Hubble Faint Object Camera, J. Geophys. Res., 103, 20,195, 1998.

Russell, C., and D. Huddleston, The unipolar inductor myth: Mass addition or motional electric field as the source of field-aligned currents at Io, $A d v$. Space Res., 26, 1665, 2000.

Saur, J., F. M. Neubauer, D. F. Strobel, and M. E. Summers, Three-dimensional plasma simulation of Io's interaction with the Io plasma torus, J. Geophys. Res., 104, 25,105, 1999.

Schinder, K., M. Hesse, and J. Birn, Magnetic field-aligned potentials in nonideal plasma flow, Astrophys. J., 380, 293, 1991.

Waite, J. H., Jr., T. E. Cravens, J. U. Kozyra, A. F. Nagy, S. K. Atreya, and R. H. Chen, Electron precipitation and related aeronomy of the Jovian thermosphere and ionosphere, J. Geophys. Res., 88, 6143, 1983.

Wilkinson, M. H., Evidence for periodic modulation of Jupiter's decametric radio emission, J. Geophys. Res., 103, 19,985, 1998.

Williams, D. J., R. M. Thorne, and B. Mauk, Energetic electron beams and trapped electrons at Io, J. Geophys. Res., 104, 14,739, 1999.

J. T. Clarke, Center for Space Physics, Boston University, Boston, MA 02215, USA. (jclarke@bu.edu)

P. Delamere, Laboratory for Atmospheric and Space Physics, University of Colorado, Boulder, CO 80309-0391, USA. (delamere@lasp.Colorado. edu)

J.-C. Gérard, D. Grodent, and J. Gustin, Laboratoire de Physique Atmosphérique et Planétaire, Université de Liege, Liege B-4000, Belgium. (jc.gerard@ulg.ac.be; D.Grodent@ulg.ac.be; J.Gustin@ulg.ac.be) 


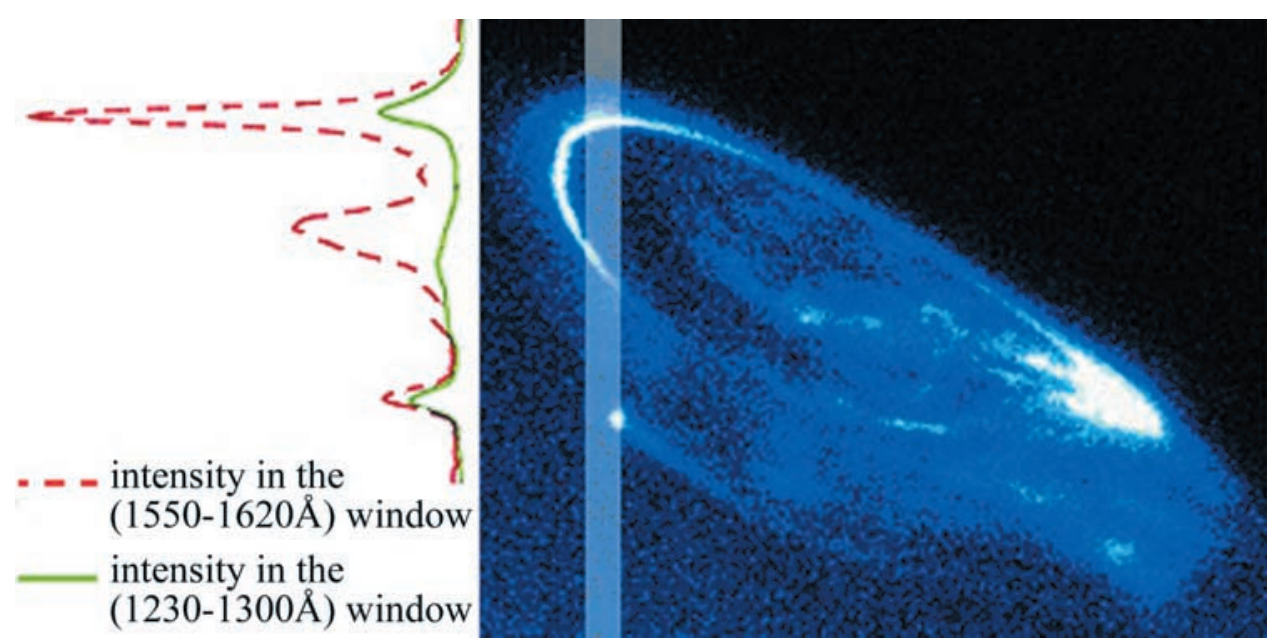

Figure 1. Geometry of the spectral observations of the Io footprint. The image was obtained on 22 February 2000 approximately $12 \mathrm{~min}$ before the G140L spectrum (case 3 in Table 1 ). The position of the STIS slit is indicated as a lighter blue line. The intensity variations measured along the slit in two spectral regions (one absorbed by methane, one not) are shown on the left. 